\title{
Choosing of Optimal Voltage Amplitude of Four Pairs Square Piezoelectric Elements for Minimization of Acoustic Radiation of Vibrating Plate
}

\author{
M. KOZIEN ${ }^{a, *}$ AND J. WICIAK ${ }^{b}$ \\ ${ }^{a}$ Institute of Applied Mechanics, Cracow University of Technology \\ al. Jana Pawła II 37, 31-864 Kraków, Poland \\ ${ }^{b}$ Department of Mechanics and Vibroacoustics, University of Science and Technology — AGH \\ al. Mickiewicza 30, 30-095 Kraków, Poland
}

\begin{abstract}
The study is a next part of earlier works by the authors, and explores how does the sequence of activated actuators and the level of applied voltage affect the radiated acoustic energy. The analysis uses the finite element method for structural vibrations and combination of the finite element and the intensity hybrid method to assess the level of sound radiation. The vibrating element is a steel plate with glued on actuators, supported on one edge and excited by a harmonically variable, concentrated load with a constant amplitude value.
\end{abstract}

PACS numbers: 43.20.Tb, 33.15.Mt, 34.50.Ez, 77.22.Gm

\section{Introduction}

Minimization of acoustic energy radiated by vibrating surface elements is still a major issue in practical applications. When the elements of the mechanical system are small enough, one of the state-of-the-art techniques of active vibro-control can be employed utilising surface piezoelectric actuators in the form of piezoceramic elements or piezoelectric foils. The piezoelectric effect is produced when voltage is applied to opposite surfaces of an element made of material displaying piezoelectric properties, inducing strains in the element. When such an element is properly mounted on the external surface of a vibrating structural element, the surface type forces are generated which are able to the dynamic characteristic of the whole system [1-4]. On account of the way forces are generated such system is easily controllable. Depending on the manner the external excitation is applied and on excitation frequency, the system response might contain one predominant mode or a superposition of several modes of various weight factors. Fuller and Elliott $[1,2]$ reported that the lowest modes are of key importance for sound radiation. The efficiency of radiation of particular modes depends on the volume of the surrounding medium within the vibrating element can move. In previous papers the influence of the curvature on sound radiation were investigated [5-8]. This study is a continuation of earlier works by the authors [9-13] and explores how the sequence of activated actuators and the level of applied voltage affect the radiated acoustic energy. In the current research programme the actuators are dispersed. The vibrating element is a steel plate with glued on actuators, supported on one edge and ex-

* corresponding author; e-mail: kozien@mech.pk.edu.pl cited by a harmonically variable, concentrated load with a constant amplitude value.

\section{Numerical analysis of the system}

Let us assume that all four pairs of actuators are supplied with the same harmonically variable voltage and that they are ideally attached to the plate surface. Accordingly, the equations of bending vibrations due to the action of piezoelectric actuators and concentrated load is written as

$$
\begin{aligned}
& \left(\frac{\partial^{4} w}{\partial x^{4}}+2 \frac{\partial^{4} w}{\partial x^{2} \partial y^{2}}+\frac{\partial^{4} w}{\partial y^{4}}\right)+\mu\left(\frac{\partial^{5} w}{\partial x^{4} \partial t}\right. \\
& \left.\quad+2 \frac{\partial^{5} w}{\partial x^{2} \partial y^{2} \partial t}+\frac{\partial^{5} w}{\partial y^{4} \partial t}\right)+\frac{\rho h}{D} \frac{\partial^{2} w}{\partial t^{2}} \\
& \quad=\frac{C_{0} \varepsilon_{\mathrm{pe}}}{D} \sum_{i=1}^{4}\left\{\left[\delta^{\prime}\left(x-x_{1 i}\right)-\delta^{\prime}\left(x-x_{2 i}\right)\right]\right. \\
& \left.\quad \times\left[H\left(y-y_{1 i}\right)-H\left(y-y_{2 i}\right)\right]\right\} \\
& \quad+\frac{C_{0} \varepsilon_{\mathrm{pe}}}{D} \sum_{i=1}^{4}\left\{\left[H\left(x-x_{1}\right)-H\left(x-x_{2}\right)\right]\right. \\
& \left.\quad \times\left[\delta^{\prime}\left(y-y_{1}\right)-\delta^{\prime}\left(y-y_{2}\right)\right]\right\}+\frac{q_{0}}{D} \mathrm{e}^{\mathrm{j} \omega t}, \\
& D=\frac{E_{\mathrm{p}} h^{3}}{12\left(1-\nu_{\mathrm{p}}^{2}\right)}, \quad \text { and } C_{0} \varepsilon_{\mathrm{pe}} \\
& =\frac{D 3 E_{\mathrm{pe}}\left[\left(h_{b}+h_{\mathrm{pe}}\right)^{2}-h_{b}^{2}\right]\left(1-\nu_{\mathrm{p}}\right)}{2 E_{\mathrm{pe}}\left[\left(h_{b}+h_{\mathrm{pe}}\right)^{3}-h_{b}^{3}\right]\left(1-\nu_{\mathrm{p}}\right)+2 E_{\mathrm{p}} h_{b}^{3}\left(1-\nu_{\mathrm{pe}}\right)} \\
& \times \frac{d_{31} V}{h_{\mathrm{pe}}}=D K^{f} \varepsilon_{\mathrm{pe}},
\end{aligned}
$$


where $q_{0}$ - amplitude of surface density of external force $\left[\mathrm{N} / \mathrm{m}^{2}\right], K^{f}$ - material geometric constant $\left[\mathrm{m}^{-1}\right], V-$ applied voltage, $h_{b}=0.5 h_{\mathrm{p}}, h_{\mathrm{pe}}$ - thickness of piezoelectric material, $E_{\mathrm{p}}-$ Young modulus of plate material $[\mathrm{Pa}], h$ - plate thickness $[\mathrm{m}], \nu_{\mathrm{p}}$ - Poisson's ratio of plate material [-], $\mu$ - damping ratio [-], $\rho_{\mathrm{p}}$ - density of plate material $\left[\mathrm{kg} / \mathrm{m}^{3}\right], F_{0}$ - amplitude of concentrated load located at $\left(x_{i}, y_{i}\right)-q_{0}(x, y)=F_{0} \delta\left(x-x_{i}\right) \delta\left(y-y_{i}\right)$. The exact solution of the formulated boundary problem with homogeneous conditions is not possible. Therefore the approximate solutions should be considered. They can be found by application of the Ritz method [14, 15] or the finite element (FEM) one.

The steel plate $100 \times 100 \times 2$ considered in the study was fixed along one edge. On its two external surfaces there were four pairs of piezoelectric actuators, integrated with the plate. The external excitation has the form of a concentrated load of a point force harmonically variable in time, with the amplitude $0.5 \mathrm{~N}$. It is applied at the mid point of the free boundary, not connected with the fixed one. The coordinate system is chosen such that its origin is located in one fixed corner of the plate, the $X$ and $Y$ axes coincide with the plate edges and the $Z$ axis is perpendicular to the plate surface. The coordinates of all points on the plate are positive.

Actuators $10 \times 10 \times 1 \mathrm{~mm}$ made from PZT- 4 material are mounted symmetrically on the plate and driven $180 \mathrm{deg}$ out of phase with respect to the force signal. The modal damping coefficient for the whole structure is assumed 0.0003 . The potential on the surface of piezoelectric elements is applied such that the upper (external) electrode be positive and the lower (internal) be zero so that vibrations induced by harmonic forces should be effectively damped by a system made of four pairs of actuators (Fig. 1).

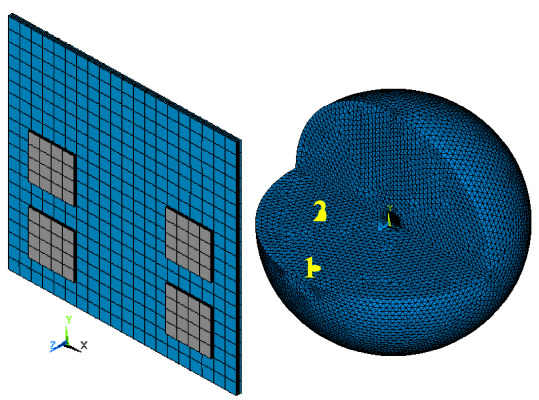

Fig. 1. FEM model of a plate with four pairs of piezoelectric actuators.

The analysis uses the FEM method for structural vibrations. The analysis uses the FEM and the intensity hybrid method to assess the level of sound radiation.

FEM analysis of plate vibrations was performed using the ANSYS package. Solid elements for coupled fields solid 45 and solid5 were chosen. Piezoelectric layers are modelled by two layers of finite elements and the steel plate - by four layers. The layer of adhesive agent was not considered in the analysis. The analysis was performed for the first four natural frequencies of vibrations of the system with piezoelectric actuators.

According to the earlier investigations [9] the characteristic configuration of the elements was chosen. For this configuration, the influence of the voltage amplitude on the reduction of the amplitude of vibrations is shown in Fig. 2.
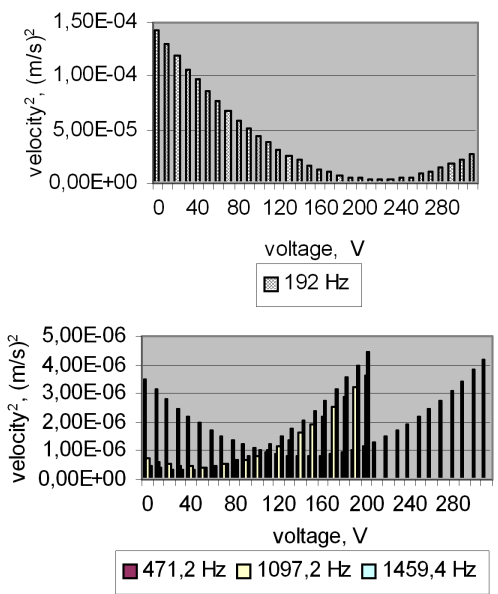

Fig. 2. The averaged square velocity in the function of applied voltage: (a) first modal frequency, (b) second, third and fourth modal frequency.

Calculations of the pressure level at chosen control points are based on the hybrid method [6]. In the hybrid method the analysis of sound radiated by vibrating surfaces consists in determining the sound intensity vector at the chosen point in the acoustic volume (space). Accordingly, the vibrating surface (i.e. the plate) is divided into sub-areas. The FEM method is applied in the analysis of structural modes. Thus obtained finite elements naturally become the sub-areas and every element is a source of radiated noise. In this case structural sounds generated by a vibrating plate were radiated to the free field.

Besides, pressure levels at the same control points were computed using the acoustic field-structure interaction module available in the ANSYS package. In the ANSYS package, the acoustic volume is modelled by solid elements of the type fluid30. Structures vibrating due to the action of mechanical sources accelerate the surrounding fluid. The normal velocity of the structure is adjusted to the normal particle velocity in the fluid. Sound radiation from the vibrating systems was simulated in a $1.1 \mathrm{~m}$ sphere (Fig. 2b) of air surrounding plates. The acoustic volume was comprised of half million 8-node elements fluid30 and infinite elements fluid130. Two kinds of acoustic elements were generated: those in contact with vibrating surface (with $u_{x}, u_{y}$, and pressure as the degrees of freedom) and those in the interior (vibrating surface, with pressure as the degrees of freedom). The mesh was finer in the plates neighbourhood. The pa- 
rameters of the air were: density $1.225 \mathrm{~kg} \mathrm{~m}^{-3}$, speed of sound $343 \mathrm{~m} \mathrm{~s}^{-1}$.

\section{Results and conclusions}

The calculation procedure was run for 15 values of harmonically varying applied voltage to piezoelectric actuator surfaces. Supported by the analysis of vibration amplitude distribution on the plate surface they would yield three voltage amplitudes: 50, 120, $220 \mathrm{~V}$. Chosen numerical results (method I) expressed as sound pressure levels are presented in Fig. 3. Acoustic pressure distributions in the examined volume for both methods were then sought for thus determined voltage levels (Table).

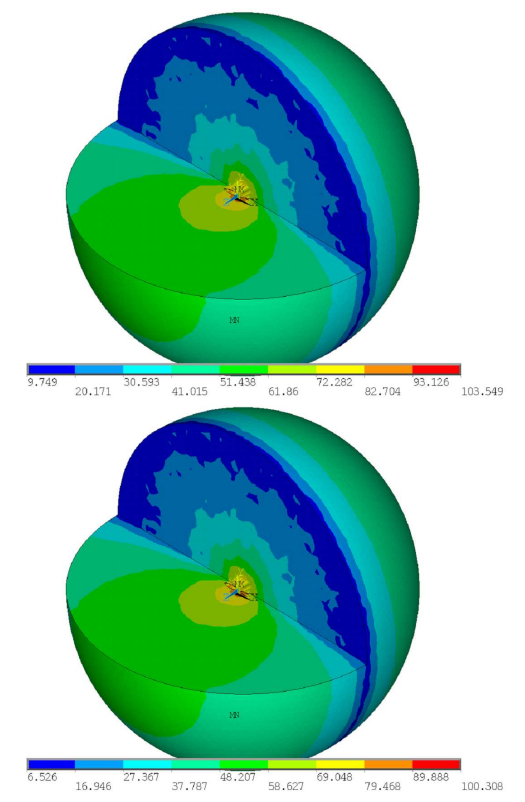

Fig. 3. Sound power lever radiated by excited plate for the lowest natural frequency: (a) without reduction, (b) with activated actuator A2.

Estimated sound pressure level.

TABLE

\begin{tabular}{|c|c|c|c|c|c|c|c|c|c|}
\hline \multirow{3}{*}{$\begin{array}{c}\text { Mode } \\
\text { No. }\end{array}$} & \multirow{3}{*}{$\begin{array}{c}\text { Freq. } \\
{[\mathrm{Hz}]}\end{array}$} & \multirow{2}{*}{\multicolumn{4}{|c|}{$\begin{array}{c}\text { Model I (FEM) } \\
\text { Sound pressure level [dB] } \\
\text { Voltage amplitude [V] }\end{array}$}} & \multirow{2}{*}{\multicolumn{4}{|c|}{$\begin{array}{c}\text { Model II (FEM + hybrid) } \\
\text { Sound pressure level [dB] } \\
\text { Voltage amplitude [V] }\end{array}$}} \\
\hline & & & & & & & & & \\
\hline & & 0 & 50 & 140 & 220 & 0 & 50 & 140 & 220 \\
\hline \multicolumn{10}{|c|}{ first control point coordinates $(x, y, z):(0.0,0.0,1.0)$} \\
\hline 1 & 192.6 & 63.0 & 60.8 & 54.9 & 47.5 & 62.3 & 60.8 & 52.6 & 46.3 \\
\hline 2 & 471.2 & 52.2 & 50.3 & 46.1 & 44.7 & 54.8 & 52.0 & 48.0 & 51.8 \\
\hline 3 & 1097.2 & 52.5 & 51.7 & 52.2 & 54.4 & 55.7 & 53.0 & 58.9 & 63.6 \\
\hline 4 & 1459.4 & 54.2 & 54.3 & 56.2 & 58.9 & 56.2 & 55.4 & 62.35 & 67.0 \\
\hline \multicolumn{10}{|c|}{ second control point coordinates $(x, y, z):(0.5,0.5,0.5)$} \\
\hline 1 & 192.6 & 63.1 & 60.9 & 54.9 & 47.6 & 62.2 & 60.7 & 52.6 & 46.3 \\
\hline 2 & 471.2 & 54.8 & 52.8 & 48.7 & 47.3 & 54.6 & 51.8 & 47.8 & 51.4 \\
\hline 3 & 1097.2 & 49.7 & 49.1 & 49.1 & 62.6 & 54.8 & 51.9 & 57.7 & 62.4 \\
\hline 4 & 1459.4 & 59.6 & 59.7 & 62.7 & 65.6 & 54.7 & 53.5 & 60.5 & 65.1 \\
\hline
\end{tabular}

The discussed numerical analysis can be summarized in the following way:
- The averaged vibration acceleration amplitude was reduced 40-, 4-, 2- and 1.5-fold for the first four modes for the precisely controlled voltage. Results reveal that structural vibrations and hence sound radiation by the given element can be vastly reduced by this method. Depending on the frequency and the applied voltage, the sound pressure level was reduced by about $15 \mathrm{~dB}$ for $192.6 \mathrm{~Hz}$ and by $8 \mathrm{~dB}$ for $471.2 \mathrm{~Hz}$.

- The major factors affecting the vibration reduction performance include the shape and actual configuration of piezoelectric elements and the amplitude of applied voltage. These parameters are associated with the mode shapes and might be optimized or controlled in active noise reduction and vibration reduction systems.

- Results of FEM analyses of sound radiation are consistent with those obtained by the hybrid method.

\section{Acknowledgments}

This study is a part of the research project 4 T07B 03429 supported by the Ministry of Science and Higher Education, Poland.

\section{References}

[1] S.P. Elliott, P.A. Nelson, Active Control of Vibrations, Academic Press, London 1997.

[2] C.R. Fuller, J. Sound Vibrat. 136, 1 (1990).

[3] T. Pustelny, B. Pustelny, Europ. Phys. J. - Special Topics 154, 265 (2008).

[4] T. Pustelny, A. Opilski, B. Pustelny, Acta Phys. Pol. A 114, A-183 (2008).

[5] S.I. Hayek, in: Fluid/Structure Interaction. Encyclopaedia of Vibration, Ed. S. Braun, D. Evins, S.S. Rao, Academic Press, Amsterdam 2002, p. 544.

[6] M.S. Kozien, J. Theor. Appl. Mech. 43, 119 (2005).

[7] M.S. Kozien, J. Nizioł, in: Proc. ICTAM04, Eds. W. Gutkowski, T.A. Kowalewski, Springer, e-book SM25 12511 (2005).

[8] M.S. Kozień, J. Wiciak, Mol. Quant. Acoust. 24, 97 (2003).

[9] M.S. Kozień, J. Wiciak, Tech. Bull. Cracow Univ. Technol. 7-M, 120 (2004).

[10] J. Nizioł, M.S. Kozień, J. Theor. Appl. Mech. 38, 351 (2000).

[11] A. Tylikowski, in: Damping of Vibrations, Ed. Z. Osiński, PWN, Warszawa 1997, p. 113.

[12] J. Wiciak, Arch. Acoust. 31, 503 (2006).

[13] A. Szpakowski, C. Tyszkiewicz, T. Pustelny, Acta Phys. Pol. A 114, A-239 (2008).

[14] J. Wiciak, Europ. Phys. J. - Special Topics 154, 229 (2008).

[15] D. Young, J. Appl. Mech. 17, 448 (1950). 